\title{
Kinetic model of electron transport in cylindrical nanowire with rough surface
}

\author{
S. A. Botman, S. B. Leble \\ Immanuel Kant Baltic Federal University, ul. Aleksandra Nevskogo, 14, Kaliningrad, 236016, Russia \\ sbotman@innopark.kantiana.ru, sleble@kantiana.ru
}

PACS 03.65.Nk, 05.60.-k

DOI 10.17586/2220-8054-2018-9-2-206-211

\begin{abstract}
In this work, the problem of electron transport in cylindrical nanowires is considered. A model of nanowire is proposed with the irregularities/scatterers concentrated mainly in the vicinity of the surface. It is treated as a waveguide with some scattering indicatrix introduced to describe specular and nonspecular scattering. Employing the kinetic approach, Kolmogorov equation is used to calculate subsequently aproximate nonequlibrium distribution function and derive explicit formula for the resistivity of the system.
\end{abstract}

Keywords: resistivity, kinetic equation, scattering.

Received: 5 January 2018

Revised: 29 January 2018

\section{Introduction}

One of the important feature of low dimensional systems is that they can exhibit properties which differ markedly from the corresponding bulk systems [1]. The problem of transport in these systems has commanded great attention over the past decades from both experimental and theoretical physicists [2-5]. Although numerous approaches to the electron transport modeling problem were developed, most of them rely on Kolmogorov equation [6] in the view of the fact that conductivity process is a nonequlibrium one.

The key component of kinetic approach is collision integral determination, which can be done in various ways. An expression for the integral can be obtained directly by calculating Bloch waves scattering amplitudes [7,8] or by introducing some semiempirical scattering indicatrix. Fuchs proposed model for DC conductivity in thin metal films based on combination of specular and nonspecular electron scattering on film boundary [9]. This approach was adopted and enhanced in a number of works [10-12].

The main goal of this work is to study Kolmogorov equation for conductivity electron within rough surface cylindrical nanowire with certain type of scattering indicatrix implied. In contrast with multiple scattering expansion, we use an expansion with respect to a small parameter [1] characterizing a weak deviation from equilibrium that leads to conventional Fredholm integral equations of convolution type. Its investigation gives simple solutions obtained using Fourier transform.

\section{Kinetic model}

\subsection{Scattering models}

Let's consider electron transport problem for cylindrical nanowire. As a source of electrons, we take the base of the cylinder, i.e. a round plate, that emits the electrons distributed by Fermi-Dirac function. Resistivity of such a system arises from the scattering of the electrons on phonons, other electrons or structural defects. For low-dimensional structures, the surface can be treated as defect and is believed to have biggest contribution to resistivity at low temperatures [3].

It is natural to introduce cylindrical coordinate system $(\rho, \phi, z)$ with respect to the symmetry of the system so that axis $z$ is aligned with the center of cylinder. Also, one may introduce cylindrical coordinate $\operatorname{system}\left(v_{\rho}, v_{\phi}, v_{z}\right)$ in velocity subspace of phase space. Assuming orts in velocity subspace are denoted as $\left(\vec{e}_{\rho}, \vec{e}_{\phi}, \vec{e}_{z}\right)$, the confinement condition for scattered electron can be expressed as follows:

$$
\left(\overrightarrow{v^{\prime}}, \vec{e}_{\rho}\right) \leq 0
$$

where $\overrightarrow{v^{\prime}}$ - velocity of electron after scattering on surface.

Generally, if the precise scattering cross section $\Sigma\left(\vec{v}, \overrightarrow{v^{\prime}}, \vec{r}\right)$ is known, it can be reduced to averaged cross section:

$$
\sigma\left(\vec{v}, \overrightarrow{v^{\prime}}, \rho\right)=\iint \Sigma\left(\vec{v}, \overrightarrow{v^{\prime}}, \vec{r}\right) d z d \phi
$$




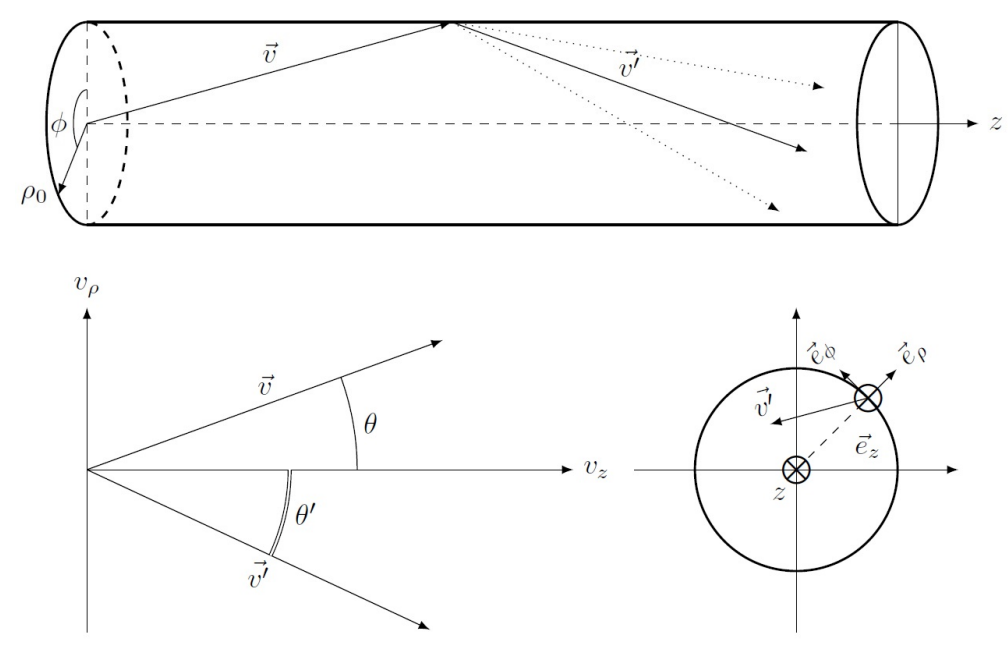

FIG. 1. Electron scattering on rough boundary of cylindrical nanowire introduced (top). Velocities of electron before and after scattering in velocity subspace (bottom left). Illustration of confinement condition (bottom right)

However, the problem of finding exact form of $\Sigma$ is of immense complexity. That is why the usual approach is to directly introduce semiempirical averaged scattering cross section $\sigma$, such as specular reflection and elastic random angle scattering [12].

The differential cross section for elastic specular scattering on a boundary will have the following form:

$$
\sigma=\delta\left(\rho-\rho_{0}\right) \delta\left(v_{z}^{\prime}-v_{z}\right) \delta\left(v_{\rho}^{\prime}-v_{\rho}\right) \delta\left(v_{\phi}^{\prime}-v_{\phi}-\pi\right) .
$$

Assuming that the nanowire is homogeneous and scattering cross section does not dependent upon the position on the surface, one may drop $\phi$ coordinate (with respective velocity component) from consideration, so the remaining coordinates are $\rho \in[0, \infty), z \in(-\infty, \infty)$ and the problem turns into a two-dimensional one. Thus, for elastic the scattering of an electron on a perfectly smooth boundary $\left(v_{z}^{\prime}=v_{z}, v_{\rho}^{\prime}=-v_{\rho}\right)$, the differential cross section appears as follows:

$$
\sigma=\delta\left(\rho-\rho_{0}\right) \delta\left(v_{z}^{\prime}-v_{z}\right) \delta\left(v_{\rho}^{\prime}+v_{\rho}\right) \Theta\left(-v_{\rho}^{\prime}\right)
$$

where $\rho_{0}$ - nanowire radius, $\delta$ - Dirac delta function, $\Theta$ - Heaviside step function. The latter multiplier in (4) is the confinement condition (1).

The main difference of rough boundary with smooth one is that the velocity angle of scattered particle follows some distribution. However, preserving the condition of elastic scattering conserves the velocity modulus. In order to take into account this conservation law, one may introduce the velocity modulus $v$ and velocity direction $\theta$ (assuming that $\theta=0$ corresponds to direction along $z$-axis). Thus, for rough wall scattering, the differential cross section will have the following form:

$$
\sigma=\delta\left(\rho-\rho_{0}\right) \delta\left(v^{\prime}-v\right) G\left(\theta^{\prime}+\theta\right) \Theta\left(-\theta^{\prime}\right) \Theta\left(\theta^{\prime}+\pi\right),
$$

where $G$ - some distribution function. The sum within brackets of $G$ implies that the maximum scattering probability corresponds to the case when the incident angle equals the scattering angle.

Or, in more general case, taking into account extent of the outer surface, one may transform (5) as follows:

$$
\sigma=A(\rho) \delta\left(v^{\prime}-v\right) G\left(\theta^{\prime}+\theta\right) \Theta\left(-\theta^{\prime}\right) \Theta\left(\theta^{\prime}+\pi\right),
$$

where $A(\rho)$ - some radial distribution of scatterers (one may choose $F(\rho)=\Theta\left(\rho_{0}-\rho\right)$ in case of infinitely thin surface layer). and $G$ - some functions, that describe scattering probability with respect to angles.

\subsection{Kinetic equation}

Let's now consider the kinetic problem for electrons moving in a cylindrical nanowire with an applied electrostatic field. In the simplest case, for transport studies we assume that the external electrical field with intensity $E$ is applied along the $z$-axis and system is finite and restrained by electrode contacts from both sides.

The Kolmogorov equation for this case will have the following form:

$$
\frac{\partial f}{\partial t}+e E \frac{\partial f}{\partial v_{z}}+v_{z} \frac{\partial f}{\partial z}+v_{\rho} \frac{\partial f}{\partial \rho}+v_{\phi} \frac{\partial f}{\partial \phi}=-f \int \sigma\left(r, \vec{v}, \overrightarrow{v^{\prime}}\right) d \overrightarrow{v^{\prime}}+\int \sigma\left(r, \overrightarrow{v^{\prime}}, \vec{v}\right) f\left(\vec{v}=\overrightarrow{v^{\prime}}\right) d \overrightarrow{v^{\prime}}
$$


where, $f=f(t, \vec{r}, \vec{v})$ - distribution function, $\sigma=\sigma\left(\vec{r}, \vec{v}, \overrightarrow{v^{\prime}}\right)$ - scattering cross section, $e$ - elementary electric charge.

Electrons on cathode follow the Fermi-Dirac distribution:

$$
n_{F}(v)=\left[\exp \left(\frac{m v^{2} / 2-E_{F}}{k_{B} T}\right)+1\right]^{-1},
$$

where $T$ - temperature, $k_{B}$ - Boltzmann constant, $E_{F}$ - Fermi energy, $m$ - electron mass.

Next, it is safe to assume that equilibrium distribution function can be chosen as follows:

$$
f_{e}=n_{F}(v) F(\rho)
$$

where $F(\rho)$ - some radial distribution of charge carriers (one may choose $F(\rho)=\Theta\left(\rho_{0}-\rho\right)$ in case of infinitely thin surface layer).

Henceforward, we will consider the simplest case of stationary $(\partial f / \partial t=0)$ and homogeneous $(\partial f / \partial z=0$, $\partial f / \partial \phi=0)$ electron current. In this case, equation (7) turns into:

$$
e E \frac{\partial f}{\partial v_{z}}+v_{\rho} \frac{\partial f}{\partial \rho}=-f \int \sigma\left(\rho, \vec{v}, \overrightarrow{v^{\prime}}\right) d \overrightarrow{v^{\prime}}+\int \sigma\left(\rho, \vec{v}, \overrightarrow{v^{\prime}}\right) f\left(\vec{v}=\overrightarrow{v^{\prime}}\right) d \overrightarrow{v^{\prime}}
$$

where $f=f\left(\rho, v_{\rho}, v_{z}\right)$ (phase space of the system is reduced to three dimensions).

Let's assume that deviation $f_{d}$ of nonequilibrium distribution function $f$ from equilibrium one $f_{e}$ is small, i.e. $f=f_{e}+\varepsilon f_{d}$, where we introduced $\varepsilon-$ small parameter $(|\varepsilon| \ll 1)$.

It should be noted that substitution of $\vec{v}$-symmetric function $f_{e}$ into right part of (10) gives zero. Considering this fact, and taking into account that $f_{d}$ is $\vec{v}$-antisymmetric, putting for brevity $\epsilon=1$, one finally obtains:

$$
e E \frac{\partial f_{e}}{\partial v_{z}}+v_{\rho} \frac{\partial f_{e}}{\partial \rho}=-f_{d} \int \sigma\left(\rho, \vec{v}, \overrightarrow{v^{\prime}}\right) d \overrightarrow{v^{\prime}}+\int \sigma\left(\rho, \vec{v}, \overrightarrow{v^{\prime}}\right) f_{d}\left(\vec{v}=\overrightarrow{v^{\prime}}\right) d \overrightarrow{v^{\prime}} .
$$

\subsection{Rough boundary case}

Let's now consider more general case of the rough boundary. This means a reflection angle is not equal to incident one, though scattering is elastic and velocity module is preserved. For this case, we substitute (6) into (11) and switch to integration over $v^{\prime}$ and $\phi^{\prime}$. In this case, the equation (11) will have the following form:

$$
e E \frac{\partial f_{e}}{\partial v_{z}}+v_{\rho} \frac{\partial f_{e}}{\partial \rho}=-f_{d}(\rho, v, \theta) A(\rho) v \int_{-\pi}^{0} G\left(\theta^{\prime}+\theta\right) d \theta^{\prime}+A(\rho) v \int_{-\pi}^{0} G\left(\theta^{\prime}+\theta\right) f_{d}\left(\rho, v, \theta^{\prime}\right) d \theta^{\prime} .
$$

Next, we introduce the integral operator:

$$
\begin{gathered}
\hat{K} f(\rho, v, \theta)=\int_{-\pi}^{0} G\left(\theta^{\prime}+\theta\right) f_{d}\left(\rho, v, \theta^{\prime}\right) d \theta^{\prime}, \\
g=-\frac{e E F(\rho) \frac{\partial n_{F}}{\partial v_{z}}+v_{\rho} n_{F} \frac{\partial F(\rho)}{\partial \rho}}{A(\rho) v},
\end{gathered}
$$

where we took into account probability normalization condition $\int_{-\pi}^{0} G\left(\theta^{\prime}+\theta\right) d \theta^{\prime}=1$.

Thus, (12) turns into:

$$
f_{d}=g+\hat{K} f_{d}
$$

which in fact is a Fredholm equation of the second kind. It is useful to introduce a new variable $\alpha=-\theta^{\prime}$ so that the kernel of integral equation $G(\theta-\alpha)$ is defined on the square i.e. $\theta, \alpha \in[0, \pi]$.

\subsection{Fourier transform solution}

In order to study equation (15), lets rewrite it in more general form:

$$
\varphi=g+\lambda \hat{K} \varphi
$$

where $\varphi$ - unknown function, $\hat{K}$ - integral operator of convolution type, $g$ - nonhomogeneous term, $\lambda$-parameter:

$$
\varphi(x)=\lambda \int K(x-s) \varphi(s) d s+g(x) .
$$


Here, integration is performed over an infinite region and function $K$ is defined by extending $G$ with zero outside $[0, \pi]$ intervals.

In general form, solution of (16) can be expressed as follows via the resolvent:

$$
\varphi=(1-\lambda \hat{K})^{-1} g
$$

In order to check for the existence of the solution (18) one may follow the Fredholm alternative and solve the corresponding homogeneous equation:

$$
\varphi=\lambda \hat{K} \varphi
$$

Let us apply Fourier transform to each element of equation (19):

$$
\begin{gathered}
\varphi=\frac{1}{\sqrt{2 \pi}} \int \tilde{\varphi}\left(\omega_{1}\right) e^{i \omega_{1} s} d \omega_{1}, \\
K(x-s)=\frac{1}{\sqrt{2 \pi}} \int \tilde{K}\left(\omega_{2}\right) e^{i \omega_{2}(x-s)} d \omega_{2} .
\end{gathered}
$$

Thus:

$$
\int \tilde{\varphi}(\omega) e^{i \omega x} d \omega=\frac{\lambda}{\sqrt{2 \pi}} \iiint \tilde{K}\left(\omega_{2}\right) e^{i \omega_{2}(x-s)} \tilde{\varphi}\left(\omega_{1}\right) e^{i \omega_{1} s} d \omega_{1} d \omega_{2} d s
$$

Let's multiply the left and right part of this equation by $e^{-i \omega_{0} x}$ and integrate over $x$ :

$$
\begin{array}{r}
\sqrt{2 \pi} \int \tilde{\varphi}(\omega) \delta\left(\omega-\omega_{0}\right) d \omega=\frac{\lambda}{\sqrt{2 \pi}} \iiint \tilde{K}\left(\omega_{2}\right) \tilde{\varphi}\left(\omega_{1}\right) e^{i\left(\omega_{1}-\omega_{2}\right) s}\left(\int e^{i\left(\omega_{2}-\omega_{0}\right) x} d x\right) d \omega_{1} d \omega_{2} d s= \\
=\lambda \iiint \tilde{K}\left(\omega_{2}\right) \tilde{\varphi}\left(\omega_{1}\right) e^{i\left(\omega_{1}-\omega_{2}\right) s} \delta\left(\omega_{2}-\omega_{0}\right) d \omega_{1} d \omega_{2} d s
\end{array}
$$

Next, the remaining integrals can be trivially taken:

$$
\begin{aligned}
\tilde{\varphi}\left(\omega_{0}\right)=\frac{\lambda}{\sqrt{2 \pi}} \iint \tilde{K}\left(\omega_{0}\right) \tilde{\varphi}\left(\omega_{1}\right) e^{i\left(\omega_{1}-\omega_{0}\right) s} d \omega_{1} d s= & \\
& =\frac{\lambda}{\sqrt{2 \pi}} \tilde{K}\left(\omega_{0}\right) \int \tilde{\varphi}\left(\omega_{1}\right) \delta\left(\omega_{1}-\omega_{0}\right) d \omega_{1}=\frac{\lambda}{\sqrt{2 \pi}} \tilde{K}\left(\omega_{0}\right) \tilde{\varphi}\left(\omega_{0}\right) .
\end{aligned}
$$

Or, changing the variables:

$$
\left(1-\frac{\lambda}{\sqrt{2 \pi}} \tilde{K}(\omega)\right) \tilde{\varphi}(\omega)=0
$$

Apparently, equation (25) will have no nontrivial solutions apart from cases when $K \sim \delta(x-s)$. Applying the Fredholm alternative to this problem (by setting $\lambda=1$ and defining $\hat{K}$ accordingly) proves that inhomogeneous equation (16) will have solution.

Using the same approach for the inhomogeneous equation gives us:

$$
\tilde{\varphi}(\omega)=\frac{\lambda}{\sqrt{2 \pi}} \tilde{K}(\omega) \tilde{\varphi}(\omega)+\tilde{g}(\omega)
$$

This equation is algebraic and thus can be solved easily:

$$
\tilde{\varphi}(\omega)=\frac{\tilde{g}(\omega)}{1-\frac{\lambda}{\sqrt{2 \pi}} \tilde{K}(\omega)}
$$

Finally, solution of equation (16) can be found by applying inverse Fourier transform to both parts of (27):

$$
\varphi(x)=\int \frac{\tilde{g}(\omega)}{\sqrt{2 \pi}-\lambda \tilde{K}(\omega)} e^{i x \omega} d \omega .
$$

Although expression (28) gives us formal solution for equation (15), its practical application is limited because as the problem of finding Fourier images $\tilde{g}$ and $\tilde{K}$ is not a trivial task. 


\subsection{Electron transport}

The solution of (15) can also be obtained as follows:

$$
f_{d}(\rho, v, \theta)=g(\rho, v, \theta)+\int_{0}^{\pi} R(\theta-\alpha) g(\rho, v, \alpha) d \alpha,
$$

where $R$ - the resolvent of integral operator (14). It can be shown using Fourier transofrm [13] that equation (29) can be expressed as follows:

$$
\tilde{f}_{d}(\omega)=[1+\tilde{R}(\omega)] \tilde{g}(\omega),
$$

The resolvent Fourier image can be found using equation (27):

$$
\tilde{R}(\omega)=\frac{\tilde{K}(\omega)}{\sqrt{2 \pi}-\tilde{K}(\omega)} .
$$

Let us now choose the extended scattering amplitude function $G$ as follows:

$$
G(x)=\frac{1}{\sqrt{2 \pi \gamma}} \exp \left(-\frac{1}{2 \gamma^{2}} x^{2}\right),
$$

which in fact is normal distribution. Here $\gamma$ - parameter which corresponds to standard deviation. Then, one may plug Fourier image of (32) into equation (31), arriving at:

$$
\tilde{R}(\omega)=\frac{2 \pi}{2 \pi-\sqrt{\gamma} \exp \left(-\frac{\gamma^{2}}{2} \omega^{2}\right)}-1 .
$$

Assuming the spread of scattering angles is small i.e. $\gamma \ll 1$, one may neglect the second term of denominator in the relation (33), that yields $\tilde{R}(\omega) \approx 0$. Thus, for the zero order approximation of nonequilibrium distribution function one may use the function $g$ :

$$
f_{d}^{0}=\frac{e E m}{k_{B} T} \frac{v_{z}}{v} \exp \left(\frac{\frac{m}{2}\left(v_{z}^{2}+v_{\rho}^{2}\right)-E_{F}}{k_{B} T}\right) n_{F}^{2} \frac{F(\rho)}{A(\rho)}-\frac{v_{\rho}}{v} \frac{1}{A(\rho)} \frac{\partial F(\rho)}{\partial \rho} n_{F} .
$$

Let's now switch velocity coordinate system as $\left(v_{z}=v \cos (\theta), v_{\rho}=v \sin (\theta)\right)$ :

$$
f_{d}^{0}=B(\rho, v) \cos (\theta)-C(\rho, v) \sin (\theta),
$$

where:

$$
\begin{gathered}
B(\rho, v)=e E \frac{m}{k_{B} T} \exp \left(\frac{\frac{m v^{2}}{2}-E_{F}}{k_{B} T}\right) n_{F}^{2}(v, T) \frac{F(\rho)}{A(\rho)}=-\frac{e E}{v} \frac{\partial n_{F}(v)}{\partial v} \frac{F(\rho)}{A(\rho)}, \\
C(\rho, v)=\frac{1}{A(\rho)} \frac{\partial F(\rho)}{\partial \rho} n_{F}(v) .
\end{gathered}
$$

Generally, current density of the waveguide can be obtained as follows:

$$
\vec{j}(\vec{r})=e \int \vec{v} f_{d}(\vec{r}, \vec{v}) d \vec{v}
$$

For $z$-projection of homogeneous current density one obtains:

$$
j_{z}(\rho)=e \int_{-\infty}^{\infty} \int_{-\infty}^{\infty} f_{d} v_{z} d v_{\rho} d v_{z}=e \int_{-\pi}^{\pi} \int_{0}^{\infty} f_{d} v^{2} \cos (\theta) d v d \theta .
$$

The total current through transverse surface can be calculated as:

$$
J=\int_{0}^{\rho_{0}} j_{z}(\rho) d \rho .
$$

Next, taking into account parity of functions, integral of the second term in (35) will give zero. The form of expression (35) allows to perform variable separation and if the exact $A(\rho)$ and $F(\rho)$ are known, radial dependence 
can be integrated out. Thus, one may obtain the temperature dependency of the system resistivity in zero order approximation:

$$
R=-\frac{1}{S_{0}}\left[\int_{0}^{\infty} v \frac{\partial n_{F}(v)}{\partial v} d v\right]^{-1}
$$

where $S_{0}$ - constant resulting from $\theta$ and $\rho$ integration:

$$
S_{0}=e^{2} \int_{0}^{\rho_{0}} \frac{F(\rho)}{A(\rho)} d \rho \int_{-\pi}^{\pi} \cos ^{2}(\theta) d \theta=\pi e^{2} \int_{0}^{\rho_{0}} \frac{F(\rho)}{A(\rho)} d \rho
$$

The integral in (41) nontrivially depends on temperature. It can be shown using the Sommerfeld expansion that:

$$
-\int_{0}^{\infty} v \frac{\partial n_{F}(v)}{\partial v} d v=\frac{1}{\sqrt{2 m}} \int_{0}^{\infty} \frac{n_{F}(E)}{\sqrt{E}} d E \approx \frac{\sqrt{E_{F}}}{2 \sqrt{2 m}}+\frac{\pi^{2}}{12} \frac{\left(k_{B} T\right)^{2}}{\sqrt{2 m E_{F}^{3}}}
$$

where Fermi energy also depends on temperature: $E_{F}(T) \approx E_{F}(0)\left(1-\frac{\pi^{2}}{12} \frac{\left(k_{B} T\right)^{2}}{E_{F}^{2}(0)}\right)$, where $E_{F}(0)$ depens only on fundamental constants [14].

\section{Results and discussion}

It was shown that Kolmogorov equation for electrons in cylindrical nanowire with surface scattering introduced can be conceived of as Fredholm equation of the second kind. Existence of a solution was proved by applying Fredholm alternative in the Resolvent form, that is approved by transition to the Fourier transformed equation. Zero order approximation of solution was illustrated by the case of a gently sloping surface irregularities used to study resistivity temperature dependence of system under consideration in explicit form.

\section{References}

[1] Dresselhaus M. S. et al. Springer Handbook of Nanotechnology. Springer, 2010

[2] Dingle R. B. The electrical conductivity of thin wires. Proceedings of the Royal Society of London. Series A, Mathematical and Physical Sciences, 1950, 201(1067), P. 545-560.

[3] Lin Y., Sun X., and Dresselhaus M. S. Theoretical investigation of thermoelectric transport properties of cylindrical Bi nanowires. Physical Review B, 2000, 62(7), P. 4610-4623.

[4] Pavlov B. S. and Strepetov A. V. Exactly solvable model of electron scattering by an inhomogeneity in a thin conductor Theoretical and Mathematical Physics, 1992, 90(2), P. 152-156.

[5] Leble S. B. Kolmogorov equation for Bloch electrons and electrical resistivity models for nanowires. Nanosystems: Physics, Chemistry, Mathematics, 2017, 8(2), P. 247-259.

[6] Kolmogoroff A. Über die analytischen Methoden in der Wahrscheinlichkeitsrechnung. Mathematische Annalen, 1931, 104(1), P. 415-458.

[7] Lobanov I. S., Popov I. Yu. Scattering by a junction of zig-zag and armchair nanotubes. Nanosystems: Physics, Chemistry, Mathematics, 2012, 3(2), P. 6-28.

[8] Botman S. A. and Leble S. B. Electrical conductivity model for quasi-one-dimensional structures. Nanosystems: Physics, Chemistry, Mathematics, 2017, 8(2), P. 231-235.

[9] Fuchs K. The conductivity of thin metallic films according to the electron theory of metals. In Mathematical Proceedings of the Cambridge Philosophical Society, 1938, 34(1), P. 100-108.

[10] Chaplik A. V. and Entin M. V. Energy spectrum and electron mobility in a thin film with non-ideal boundary. Soviet Journal of Experimental and Theoretical Physics, 1969, 28(3), P. 514-517.

[11] Krokhin A., Makarov N. M., and Yampolskii V. A. Theory of the surface scattering of electrons in metals with gently sloping surface irregularities. Soviet Physics-JETP, 1991, 72(2), P. 289-294.

[12] Makarov N. M., Moroz A. V., and Yampolskii V. A. Classical and quantum size effects in electron conductivity of films with rough boundaries. Physical Review B, 1995, 52(8), P. 6087-6101.

[13] Manjirov A. V. and Polyanin A. D. Reference book on integral equation. Factorial press, 2000.

[14] Ashcroft N., Mermin N., Wei D. Solid State Physics: Revised Edition. Amazon press, 2016. 